\title{
LA ARQUITECTURA ESCOLAR DE MARTORELL, BOHIGAS Y MACKAY. EL MODELO CONCENTRADO COMO OPORTUNIDAD EDUCATIVA
}

\author{
Isabel Durá Gúrpide
}

En un momento de importante reformulación del edificio escolar, los arquitectos Martorell, Bohigas y Mackay definieron un modelo escolar de gran riqueza espacial, con una gran capacidad didáctica y especial énfasis social. Esto fue facilitado por su amplio conocimiento de experiencias destacadas, la colaboración con expertos en la enseñanza y su carácter crítico e inconformista. Sus escuelas fueron unos de los ejemplos más destacados realizados en los años 60 en España y consiguieron un importante reconocimiento internacional. El estudio de estos edificios permite no sólo poner en relieve su valor patrimonial sino también recuperarlos como referencia en la actualidad.

Palabras clave: edificios escolares, historia de la arquitectura, siglo XX, España

Keywords: school buildings, History of Architecture, 20th Century, Spain

\section{INTRODUCCIÓN}

De acuerdo con el compromiso social que ha caracterizado la trayectoria de los arquitectos Josep Martorell, Oriol Bohigas y David Mackay, la educación de los más pequeños ha supuesto una de sus continuas preocupaciones; inquietud que se ha visto reflejada en sus edificios ${ }^{1}$. Su obra escolar, siempre atenta de las innovaciones en la materia, ha reflejado el desarrollo de la tipología y, con especial acento, la intensa etapa de transformación que experimentó el edificio escolar en los años 50 y $60^{2}$. Estos arquitectos participaron activamente en el estudio del edificio escolar, tanto con sus obras como con sus artículos, que reflejaron su amplio conocimiento sobre el tema y su carácter crítico, inconformista e, incluso, visionario.

Desde comienzos del siglo XX, la autoridad municipal de Barcelona había estado en la primera línea de la educación en Europa. Entre sus méritos había destacado una importante labor de investigación en educación y la creación temprana de escuelas al aire libre, centros de educación colectiva para vacaciones y escuelas comprensivas mixtas. Después de la Guerra Civil, el control de las escuelas municipales se traspasó al gobierno franquista, con el consecuente cambio de orientación en su enseñanza. No obstante, algunos de los profesores que habían sido apartados del sistema de enseñanza pública fundaron instituciones privadas para continuar con la pedagogía progresista de las antiguas escuelas municipales.

Martorell, Bohigas y Mackay participaron en la construcción de algunos de estos centros. Los encargos fueron propiciados por su proximidad a Artur Martorell, padre de Josep Martorell y uno de los principales promotores de la renovación pedagógica en Cataluña. Esta condición les permitió relacionarse con los mejores profesionales de la enseñanza y conocer los nuevos métodos pedagógicos. Al mismo tiempo, siguieron de cerca la vanguardia internacional en el campo de la arquitectura y, de manera particular, el de la edificación escolar. Sus centros educativos fueron difundidos a través de publicaciones y llegaron a convertirse en un referente.

En base a esto, el presente estudio analiza la obra escolar del estudio catalán con el objetivo de determinar sus características y aportaciones significativas al progreso de la edificación escolar. Para ello, se sitúa su obra en su contexto, se analizan sus edificios escolares y el discurso teórico de sus escritos. Asimismo, se pone en relación la producción escolar de estos arquitectos con la vanguardia en la materia para determinar sus aportaciones. Se emplean como fuentes principales documentación original de las obras, publicaciones de arquitectura de la época y el testimonio de Oriol Bohigas, entrevistado por la autora. En definitiva, el artículo pretende revelar las aportaciones de Martorell, Bohigas y Mackay a la importante etapa de transformación que experimentó el edificio escolar en torno a las décadas de 1950 y 1960.
1. Su preocupación sobre la educación está presente en diferentes libros de Bohigas como: Cartes de la baralla: epistolari públic sobre cultura i política, Columna, Barcelona, 2003; Oriol Bohigas: realismo, urbanidad y fracasos, T6, Pamplona, 2003; Epistolario: 1951-1994, Colegio Oficial de Aparejadores y Arquitectos Técnicos de la Región de Murcia, Murcia, 2005. Incluso, recientemente se ha publicado una entrevista a Bohigas en el diario El País, "La gente tiene que protestar para que se mejoren las ciudades", en el que vuelve ha hablar de la educación (16 de marzo de 2012).

2. La arquitectura escolar construida en España en el período 1956-1972 ha sido estudiada en profundidad en la tesis doctoral: DURÁ GÚRPIDE, Isabel, La Construcción de la Escuela Activa en España, 1956-1972, tesis doctoral (inédita), Universidad de Navarra, 2013. 
3. Algunos de los investigadores que han tratado el tema en Europa son: Herman Hertzberger y Dolf Broekhuizen en Holanda, Marco Di Nallo y Andrea Wiegelmann en Suiza, Gerain Franklin y Mark Dudek en Inglaterra, y Francisco Burgos Ruiz e Isabel Durá Gúrpide -autora del presente artículo- en España. Además, cabe destacar el VIII Congreso de la Fundación Docomomo Ibérico celebrado en Málaga en 2013 dedicado a la "Arquitectura del movimiento moderno y la educación" como muestra del interés despertado por estos edificios.

4. En la actualidad, la autora del artículo desarrolla una investigación posdoctoral sobre arquitectura escolar argentina condición que le ha permitido conocer el creciente interés sobre el tema en Argentina y en otros países de Latinoamérica. Algunos de los investigadores sobre el tema son: Francisco Ramírez Potes y Rafael Maldonado Tapias en Colombia, Carlos Ortega Ibarra y Lucía Santa Ana Lozada en México, Marcos Bencostta en Brasil, y Daniela Cattaneo y Lucía Espinoza en Argentina. También cabe citar el trabajo desarrollado por el grupo de investigación FORM de la Universidad Politécnica de Cataluña y en colaboración con distintas universidades latinoamericanas que en 2013 dedicó un número de su colección de Documentos de Arquitectura Moderna en América Latina (1950-1965) a la arquitectura docente de Brasil, Chile y México.

5. En 1951, Martorell y Bohigas obtienen el título de arquitectos por la Escuela de Arquitectura de Barcelona y se asocian profesionalmente.

6. Martorell y Bohigas se refieren a esto en sus artículos "La evolución de la tipología escolar en Catalunya" y "La escuela viva: un problema arquitectónico" respectivamente, publicados en: BOHIGAS, Oriol, MARTORELL, Josep María, MACKAY, David, Temas de arquitectura escolar, separata de la revista Cuadernos de Arquitectura y Urbanismo, BarceIona, 1972.

7. BOHIGAS, Oriol, "Carta de Oriol Bohigas a Montserrat Palmer (Barcelona, 5 de diciembre de 1962)", en Epistolario 1951-1994, cit., pp. 74-75. Bohigas trató este tema en profundidad en su artículo "Cap a una arquitectura realista", 1962.

8. BOHIGAS, Oriol, "La escuela viva: un problema arquitectónico", cit.

9. Martorell se refirió a la publicación de Uberto Siola: Tipología e architettura della scuola, Edizioni Scientifiche Italiane, Napoles, 1966. Cfr. MARTORELL, Josep María, "La evolución de la tipología escolar en Catalunya", en BOHIGAS, O., MARTORELL, J. M. y MACKAY, D., Temas de arquitectura escolar, cit., pp. 30-43.
Cabe destacar el actual interés por el tema de la arquitectura escolar. En los últimos años, han proliferado estudios que ponen en valor la arquitectura escolar de mediados del siglo veinte de distintos países europeos como Holanda, Suiza, Reino Unido y España ${ }^{3}$. En Latinoamérica, también se aprecia un aumento de la atención a la materia con el propósito de poner en valor un patrimonio cultural poco explorado que, al mismo tiempo, contribuya al diseño actual de centros educativos en un contexto de creciente demanda ${ }^{4}$. El mismo Oriol Bohigas propone recuperar las experiencias destacadas del siglo XX para la práctica actual, en la que, con frecuencia, el diseño del edificio escolar atiende más a las exigencias mínimas marcadas por las normativas que a las necesidades educativas reales.

\section{UN CAMBIO DE MENTALIDAD: EL REALISMO CRÍTICO Y LA NUEVA ARQUITECTURA ESCOLAR}

El primer contacto de Martorell y Bohigas ${ }^{5}$ con los edificios de enseñanza había llegado en 1954, de la mano del Concurso de Institutos Laborales en el que participaron junto a Joaquín Gili y Francisco Bassó. Su propuesta recibió el tercer premio y, como consecuencia, construyeron los institutos laborales de Amposta (1955-1957) y Sabiñánigo (1955-1958). Poco después, con la puesta en marcha del Plan Nacional de Construcciones Escolares, tuvieron la oportunidad de construir varios centros de educación primaria: las escuelas Baró de Viver y Timbaler del Bruc en Barcelona (1956-1957) y tres grupos escolares en Tarrasa en colaboración con Josep Pratmarsó (1960). Los arquitectos catalanes han reconocido la arquitectura racionalista de los años 20 y 30 como la principal referencia de estos proyectos ${ }^{6}$.

No obstante, la década de 1960 coincidiría con un cambio de mentalidad que se vería reflejado en sus obras. En 1962, el equipo se estableció como Martorell-Bohigas-Mackay Arquitectos con la incorporación del arquitecto inglés David Mackay y definieron juntos las pautas de su trabajo. Críticos al Movimiento Moderno -al que atribuían un excesivo idealismo ajeno a su entorno-, optaron por una arquitectura adaptada a las necesidades reales, a los problemas de carácter global, sociales, constructivos y económicos. Este pensamiento coincidía con el 'realismo crítico', extendido entre los intelectuales de la época. Bohigas explicaba al respecto?

\begin{abstract}
"Cada día se acusa aquí más claramente una separación fuertísima entre los formalismos que repiten las apariencias de una arquitectura de los años 20 y 30, y los que crecen en una posibilidad más realista, más de acuerdo con los tiempos que vivimos, en fin, más preocupada de la forma, más de acuerdo con los problemas reales de la sociedad que nos ha tocado vivir. Quizá más intensa que la que hace unos años separó los primeros intentos de la arquitectura moderna de los últimos residuos del tradicionalismo mal entendido".
\end{abstract}

El cambio de década coincidió también con un nuevo impulso en el desarrollo de la tipología escolar que fue proclamado desde distintos foros de debate a nivel internacional. Bohigas destacó como punto de inflexión la celebración de la XII Trienal de Milán, en 1960, que dedicó especial atención al tema de la escuela ${ }^{8}$. El arquitecto lo consideró un paso decisivo para la trasformación del edificio escolar, el punto de arranque de la sucesiva pérdida de importancia del aula y la máxima consideración pedagógica de los espacios comunes. En los años 60 el diseño del edificio escolar se caracterizaría por una atención especial a la totalidad de la acción educativa. Apoyado en el trabajo del arquitecto italiano Uberto Siola, Martorell sintetizó las principales necesidades del nuevo edificio escolar, que serían la clave de su propia arquitectura?:

1. Espacios servidos y espacios de servicio.

2. Un núcleo de actividades abierto al barrio, toman importancia los parámetros social y comunitario.

3. El aula de múltiples funciones, superando la enseñanza pasiva.

4. Escuelas de dimensión importante para que su presencia cuente como centro comunitario en el entorno urbano. 5. El aula como punto de referencia del grupo-clase, para que la socialización pretendida en una educación de acento comunitario no se convierta en un vago estado de ánimo de los alumnos.

6. La flexibilidad, entendida no tanto como posibilidad de modificación de la disposición del edificio, sino como caracterización de los espacios arquitectónicos para que sean adaptables a distintos usos, y para que provoquen la movilidad dentro de la escuela y fuercen la explosión del grupo clase y la creación de agrupaciones distintas en relación con muy diversos centros de interés.

7. Consecuentemente, los espacios intermedios concebidos y valorados como espacios pedagógicos.

8. Los itinerarios interiores al edificio-escuela valorados como posibilidad de encuentro y relación. 
En consecuencia, Martorell, Bohigas y Mackay proyectaron edificios escolares en los que los espacios de relación se convertían en los elementos focales alrededor de los cuales se desarrollaría toda la actividad de la escuela. Las aulas se abrían hacia espacios polivalentes, destinados a actividades en grupo, que a su vez se vinculaban a el área común principal. Asimismo, recuperaron la escala doméstica en el edificio escolar para ofrecer al niño un ambiente acogedor. Estas condiciones estuvieron especialmente presentes en sus escuelas proyectadas a partir de 1960 y se fueron consolidando en sus sucesivas obras.

\section{EL DESARROLLO DE UN MODELO CENTRALIZADO}

El primer edificio escolar que los arquitectos catalanes proyectaron en esta línea fue la escuela Sant Gregori para Sabadell, desarrollada en 1961 pero que no llegó a construirse. La escuela se organizaba en dos plantas, alrededor de espacios comunes polivalentes, destinados a actividades docentes y de relación. En este caso, la orientación de las aulas quedaba relegada a segundo término a favor de su vinculación con el espacio común central (Fig. 1). Sus autores han señalado como su principal referencia el pabellón escolar británico de la XII Trienal de Milán, resultado de más de una década de investigación de la oficina estatal dedicada a construcciones escolares.

Más tarde, con el encargo de la escuela Garbí, en Espluges de Llobregat, encontraron la oportunidad de concretar una escuela de esquema centralizado (1962-1967). Esta escuela fue promovida por un grupo de pedagogos según el proyecto educativo de Pere Vergés, director de la antigua Escuela del Mar de Barcelona, que fundaron 'Instituciones Pedagógicas S. A.'. La institución contó con subvenciones particulares de los propios ex-alumnos y el apoyo de una fuerte asociación de padres. El conjunto escolar incluiría parvulario, escuela primaria y secundaria para 400 alumnos. Martorell, Bohigas y Mackay desarrollaron el proyecto en colaboración con el equipo de pedagogos con el propósito de que la arquitectura contribuyese a la actividad educativa.

En la elaboración del proyecto, el principal objetivo de los arquitectos fue superar el aislamiento del aula y fomentar el sentido de comunidad. El planteamiento de la escuela contemplaba cierta analogía urbana: contaba con un espacio central comunitario, similar a una plaza, y en torno a él que se distribuían diversos ámbitos de mayor privacidad. En este caso, los arquitectos han señalado como referencias de partida la arquitectura escolar de Perkins y Will, en Estados Unidos, y las escuelas públicas británicas; edificios destacados por el diseño cuidado del aula y la vinculación secuencial de las diferentes áreas educativas, características que incorporarían las escuelas del estudio catalán.

La escuela Garbí fue diseñada en torno a un gran espacio de uso polivalente, que serviría para comer, jugar, hacer conciertos y conferencias. En este caso, se aumentaba la vinculación de los distintos espacios del centro respecto a la escuela Sant Gregori al disponer un único espacio central que relacionaba todos los niveles. En su perímetro se dispusieron en tres plantas las aulas, en los lados este y sur, y las áreas de servicio, talleres y administración, en las otras orientaciones. Las clases de párvulos se situaron en el nivel inferior, con acceso directo al jardín. Los espacios de circulación desaparecían como tales y se convertían en áreas docentes para actividades de pequeños grupos que vinculaban las aulas y el espacio central. Al sureste se construyó una piscina cubierta y un gimnasio separados de la actividad de la escuela (Figs. 2, 3 y 4).

El espacio central suponía un volumen prismático en el que se concentraba el mayor esfuerzo estructural, grandes cerchas metálicas salvaban la totalidad de su anchura y proporcionaban la máxima diafanidad para su uso polivalente. En contraste, las aulas se disponían de manera quebrada en el perímetro definiendo en el interior espacios intermedios de escala contenida y, a su vez, reduciendo en el exterior el impacto visual del edificio. En el área perimetral se optó por una estructura de vigas y pilares de hormigón armado que permitía la disposición flexible de las aulas. Tanto en las áreas intermedias como en las aulas se buscó recrear un ambiente acogedor para el niño a través del control de la escala y el empleo de materiales que aportaban calidez como el ladrillo visto, la cerámica y la madera. En con-

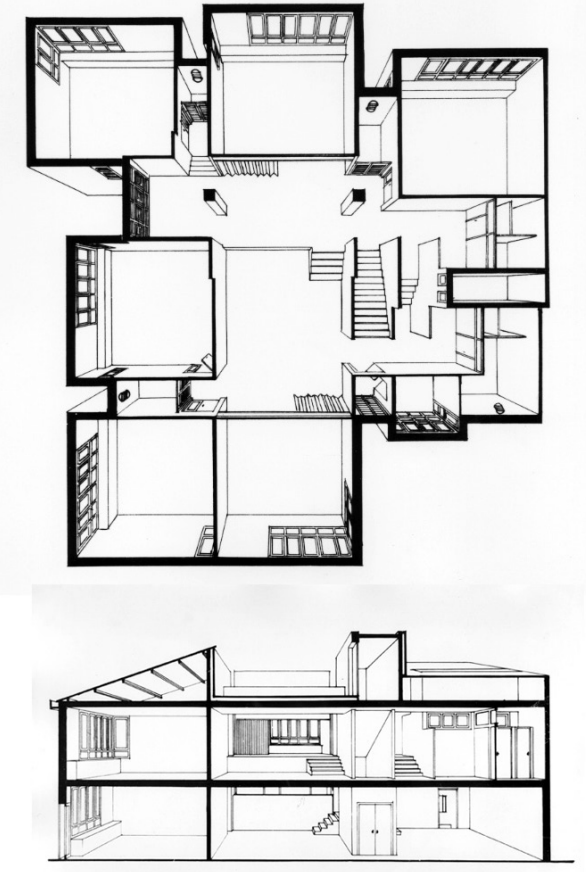

Fig. 1. Planta y sección del proyecto de la escuela Sant Gregori en Sabadell (material original facilitado por MBM Arquitectos). 


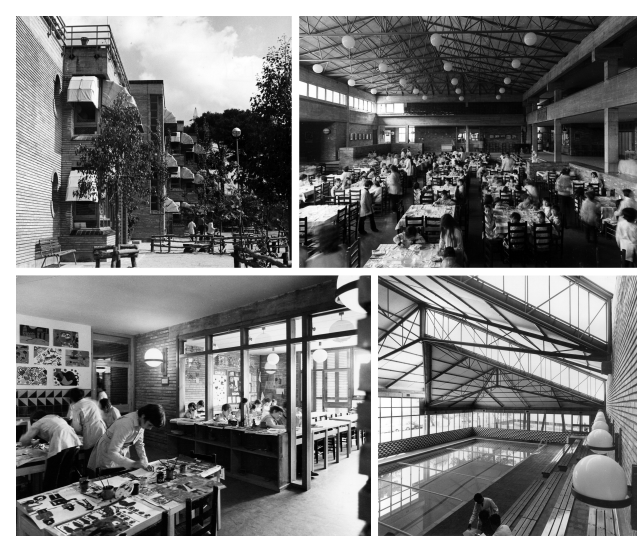

Fig. 2. Imágenes de la escuela Garbí en Esplugues de Llobregat: exterior, espacio central usado como comedor, espacios intermedios y piscina (material original facilitado por MBM Arquitectos).

Fig. 3. Sección transversal de la escuela Garbí en Esplugues de Llobregat (material original facilitado por MBM Arquitectos).

Fig. 4. Planta de la primera fase de la escuela Garbí (material original facilitado por MBM Arquitectos).

3
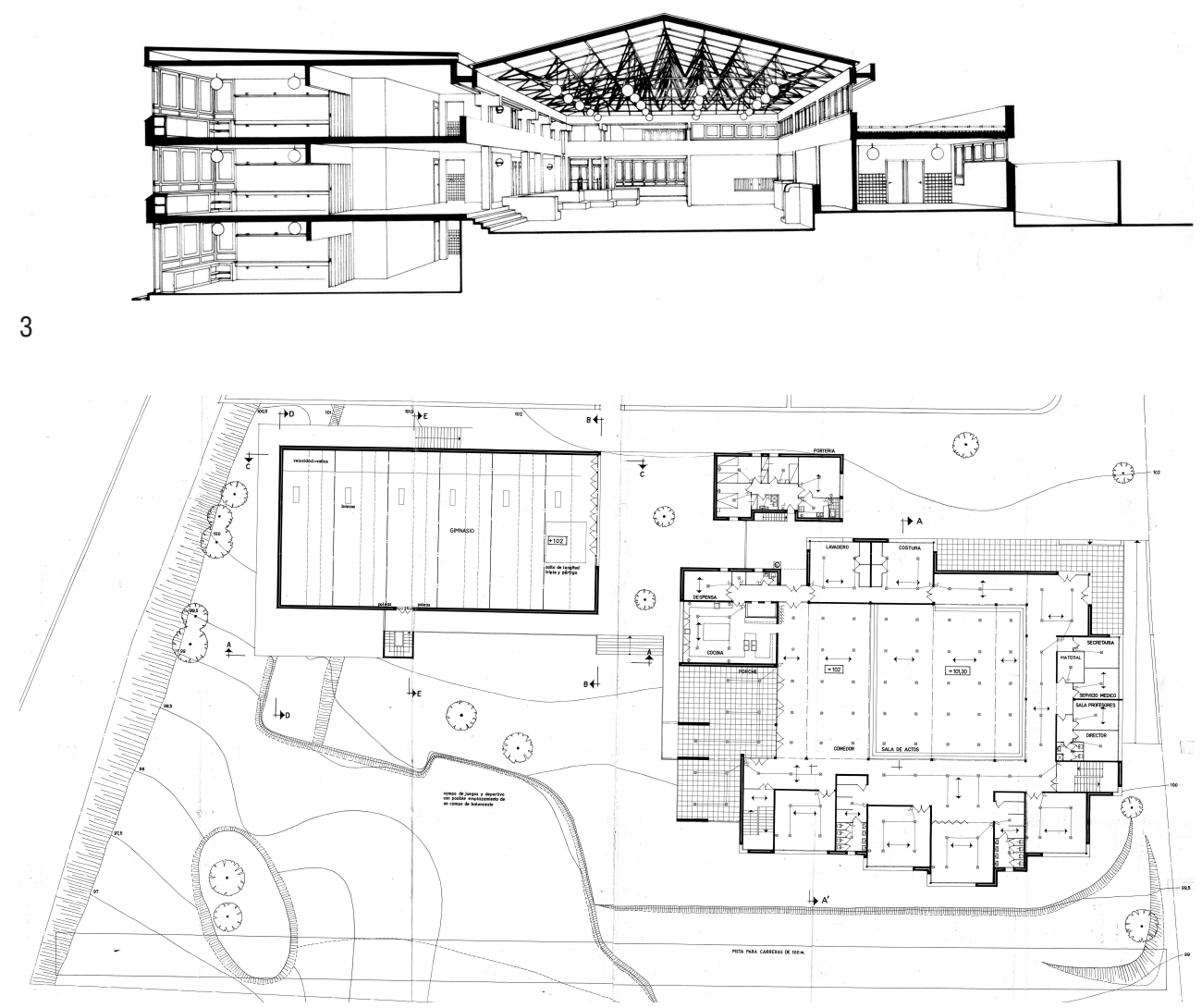

4

secuencia, cada espacio tendría su propia identidad, acorde a la condición de la actividad educativa que contenía y, a su vez, existía una vinculación fluida entre la secuencia de los distintos espacios.

Al poco tiempo de ser inaugurada la escuela, se produjeron tres cambios fundamentales que afectaron al proyecto: una nueva ordenación urbanística -que hacía necesario cambiar de lugar el acceso-, una nueva ley de educación - que alteró los programas escolares establecidos-, y la decisión del la administración de la escuela de aumentar considerablemente el número de alumnos - de 400 a 840-. Todo ello provocó una transformación y ampliación del edificio, en la que Martorell, Bohigas y Mackay trataron de no modificar el concepto inicial.

En este proceso, el espacio común con su analogía urbana se mantuvo. El criterio fue añadir elementos autónomos pero siempre aglutinando, como protagonistas espaciales, nuevas zonas de vida comunitaria. Así, mientras inicialmente la organización de la escuela era análoga a un poblado alrededor de una plaza, con su crecimiento se convertía en un conjunto a lo largo de una calle compleja y llena de posibilidades de vida social. La ampliación se realizó mediante un proceso de adhesión, posibilitado por la flexibilidad compositiva del edificio. Con estas modificaciones el proyecto perdía la radicalidad inicial pero consiguió adaptarse a las nuevas condiciones conservando sus principales virtudes (Figs. 5 y 6 ).

Esta obra tuvo un importante reconocimiento y fue publicada en revistas de arquitectura nacionales e internacionales. Rafael Moneo incluyó este edificio en su artículo "La llamada Escuela de Barcelona", publicado en la revista Arquitectura (n. 121, 1969). También tuvo presencia en otras revistas españolas como Hogares Modernos (n. 5, 1966), Nueva Forma (n. 83, 1972) y Cuadernos de Arquitectura y Urbanismo (n. 89, 1972). Asimismo, la escuela Garbí fue publicada fuera del país en L'Architettura (Roma, n. 154, 1968), Summa (Buenos Aires, n. 20, 1969), L'Architecture d'Aujourd'hui (París, n. 167, 1973), The Architectural Review (Londres, n. 961, 1977) y Werk-Architere (Zürich, n. 35-36, 1979). Esto dejaba patente el valor de la propuesta, de interés a nivel internacional, y la convirtió en una referencia para otros arquitectos. 


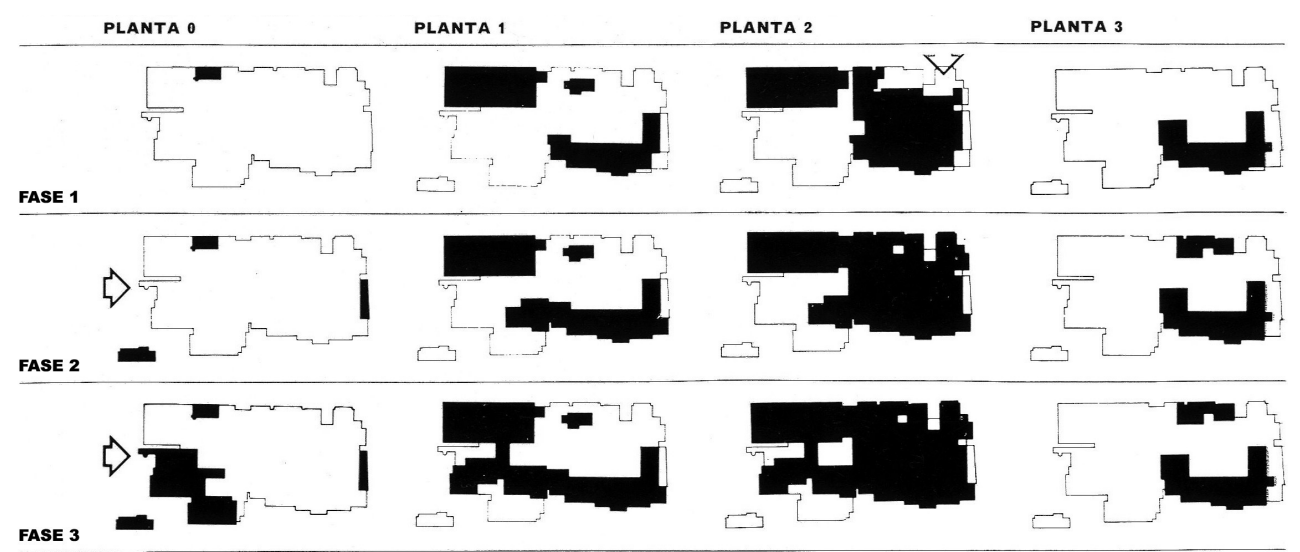

5

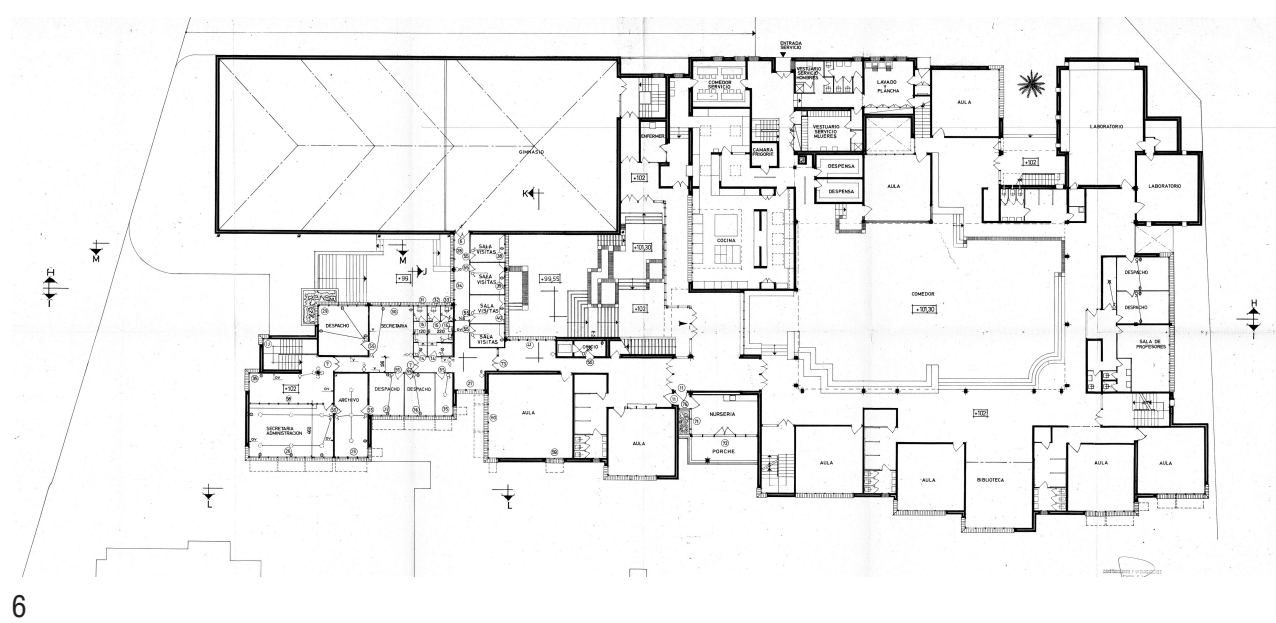

No obstante, los autores de la escuela Garbí, de acuerdo con su actitud crítica, detectaron en el proyecto cierto defecto de autosuficiencia, de cerrazón en relación con el contexto urbano y social en el cual el edificio estaba inmerso. Esta problemática fue abordada en sus siguientes edificios docentes, las escuelas promocionadas por la Caja de Ahorros de la Diputación de Barcelona. Martorell, Bohigas y Mackay proyectaron tres de estas escuelas, conocidas como Sant Jordi, en Pineda de Mar (1967-1969), Vilanova i la Geltrú (1968-1971) y Montbau (1969).

Las escuelas Sant Jordi, ideadas por el pedagogo Ramón Fuster Rabès ${ }^{10}$, tenían vocación de escuelas públicas y buscaban la apertura e interrelación con la ciudad desde de su estatuto, que establecía la corresponsabilidad de los padres del barrio o población. Así, se dispuso que una serie de servicios (biblioteca, campos de deporte, salas audiovisuales...) se situasen en el edificio de manera que pudiesen ser utilizados por los exalumnos y la gente del lugar. Esta experiencia supondría el inicio de una modalidad escolar que tendría en cuenta la formación permanente y valoraría las relaciones comunitarias que ofrecía la vida urbana como fuente educativa.

La primera de estas escuelas se situaría en la pequeña ciudad costera de Pineda de Mar, al norte de Barcelona, y contaría con guardería y enseñanza primaria para 350 alumnos. Sus requisitos fueron similares a los de la primera fase de la escuela Garbí y los arquitectos optaron por una disposición semejante. Con el propósito de resolver en esta escuela su vinculación con el vecindario, el espacio polivalente se sustituyó por varias salas especializadas con distinta disposición. Se mantuvo un área central, dividida en dos sectores, y se incorporaron una biblioteca y sala de actos en el perímetro que permitían el acceso independiente al público ajeno a la institución. Otra novedad fue la incorporación de la cubierta del edificio como extensión del patio de juego, lo que permitía aprovechar al máximo la limitada superficie del terreno (Figs. 7 y 8).
Fig. 5. Esquema de las diferentes fases de ampliación de la escuela Garbí (Cuadernos de Arquitectura y Urbanismo, n. 89,1972, p. 45).

Fig. 6. Planta de la segunda fase de la escuela Garbí (material original facilitado por MBM Arquitectos).
10. La Caja de Ahorros de la Diputación de Barcelona designó a Ramón Fuster como asesor pedagógico, defensor de la escuela de inspiración cristiana pero no confesional. Fuster proponía crear una serie de escuelas catalanas con vocación de escuelas públicas regidas por consejos escolares, precedente que marcaría la legislación después de la transición democrática. Fuster recogió sus ideas en el ensayo L'educació, problema social, Editorial Estela, Barcelona, 1965. 


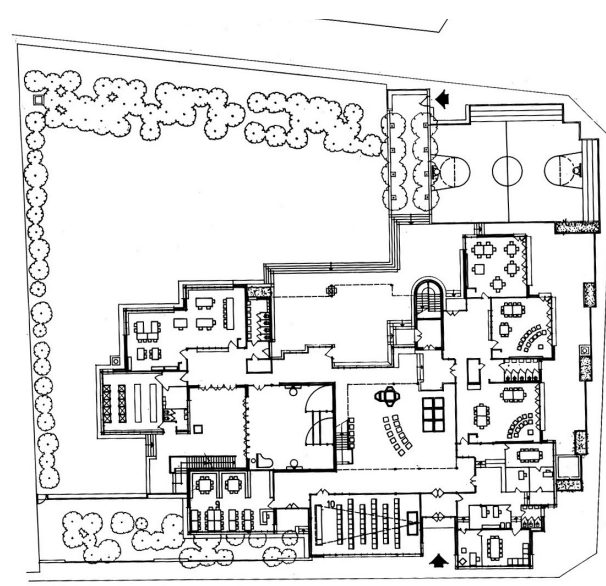

Planta baja

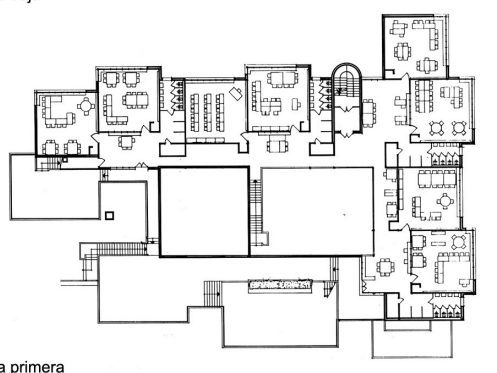

Planta primera

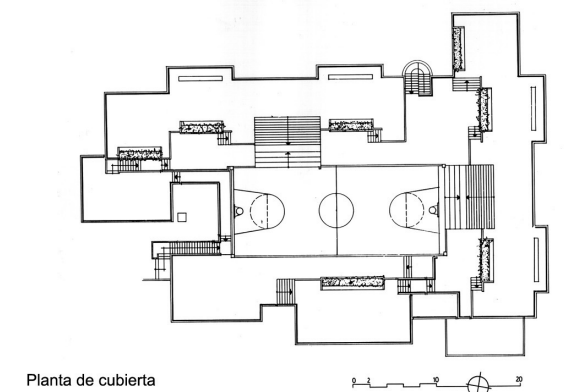

8

Fig. 7. Imágenes de la escuela Sant Jordi de Pineda de Mar: acceso, exterior desde el patio, espacios intermedios y azotea (material original facilitado por MBM Arquitectos).

Fig. 8. Plantas de la escuela Sant Jordi en Pineda de Mar (material original facilitado por MBM Arquitectos).
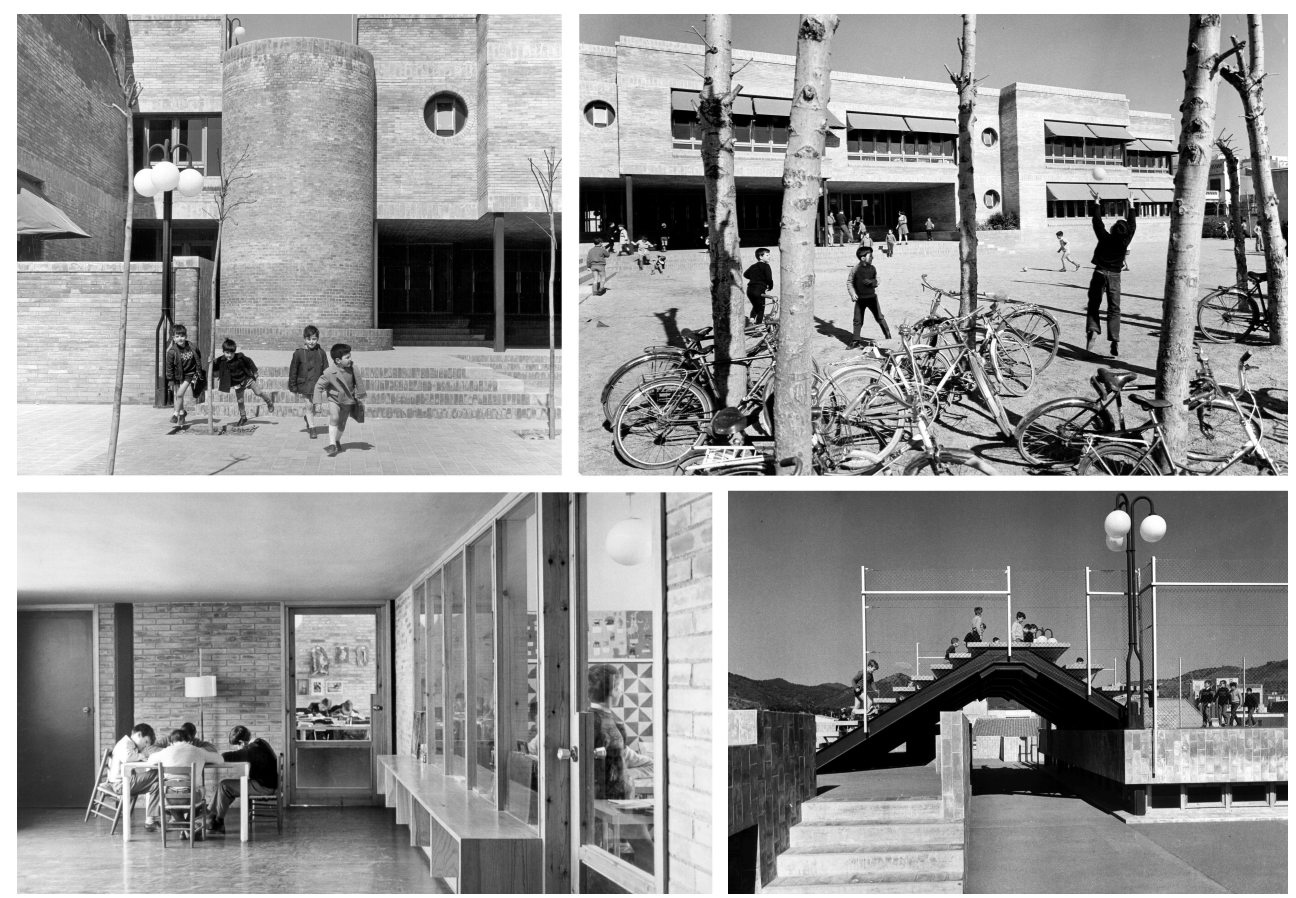

7

La escuela Sant Jordi de Vilanova i la Geltrú se destinaría a la enseñanza secundaria para 550 alumnos. A pesar del considerable aumento del número de estudiantes respecto de las escuelas anteriores, se repitió el esquema general de organización de tipo centralizado, insistiendo en la importancia del espacio principal de relación. En este caso, se mantuvo un gran espacio único en el centro y el gimnasio se situó junto a las aulas en su perímetro. No obstante, el mayor número de aulas dificultó la proximidad de éstas con el espacio central y se aumentó la superficie de las áreas intermedias. El esquema se traspasó también a la azotea donde se situó una pista deportiva en el centro y distintas áreas para actividades pedagógicas al aire libre a su alrededor (Figs. 9 y 10).

Por último, la escuela Sant Jordi de Barcelona, en el barrio de Montbau, impartiría enseñanza primaria y secundaria para un total de 560 niños y niñas. El proyecto no llegó a construirse pero supuso un eslabón importante en el desarrollo de la arquitectura escolar de sus autores ${ }^{11}$. Los arquitectos introdujeron cambios sustanciales en el esquema centralizado empleado con anterioridad para solventar los problemas que presentaba en las escuelas de gran tamaño. El edificio se organizó en cuatro volúmenes independientes que se articulaban alrededor de un espacio común al aire libre. Las diferentes partes podían funcionar con independencia y, al mismo tiempo, estaban vinculadas por una circulación helicoidal en torno al espacio central que aportaba cohesión al conjunto. Al igual que las otras escuelas Sant Jordi, el cuerpo destinado a biblioteca, sala de exposiciones y sala común contó con un acceso independiente y la cubierta se recuperó como zona de juego (Fig. 11).

En este grupo de escuelas los arquitectos emplearon las mismas estrategias de escala que en la escuela Garbí, un espacio central amplio de geometría claramente definida y aulas dispuestas en el perímetro de manera discontinua que acotaban los espacios intermedios. El sistema constructivo fue también similar: el espacio central se mantuvo diáfano aunque, en esta ocasión, se optó por cubiertas de losa reticular para permitir el tránsito de la cubierta. En el resto de la construcción se emplearon los mismos materiales. La aportación principal de estas escuelas respecto al modelo de la escuela Garbí estaba en las variaciones de la disposición del conjunto que mejoraban la relación secuencial entre los distintos espacios, mantenían la eficacia del esquema en escuelas de mayor capacidad e incorporaban la demanda de un mayor número de salas de uso común específicas.

La primera de las escuelas Sant Jordi consiguió la mayor popularidad del grupo y fue publicada en distintas revistas nacionales como Cuadernos de Arquitectura (n. 75, 1970) y
11. La escuela Costa i Llovera, promovida por la institución Propedagogic, tendría un esquema similar. El proyecto fue iniciado en 1970, de manera paralela a la escuela Sant Jordi de Montbau, pero su construcción se pospuso hasta 1976 1979. El proyecto construido asumiría algunos cambios ensayados en la escuela Thau, como los materiales, y aumentaría la independencia de sus partes. 

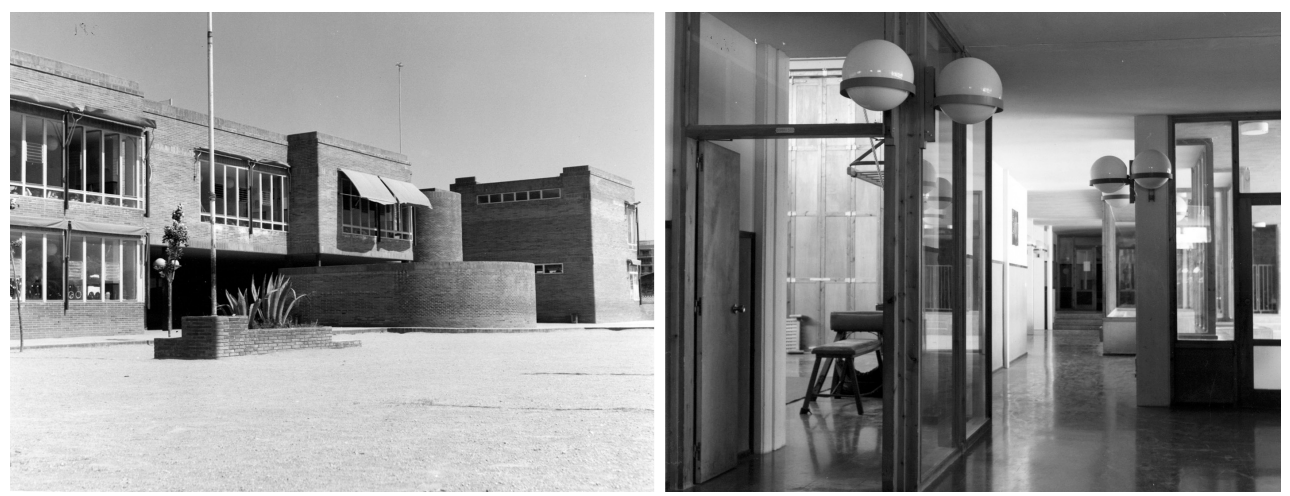

9

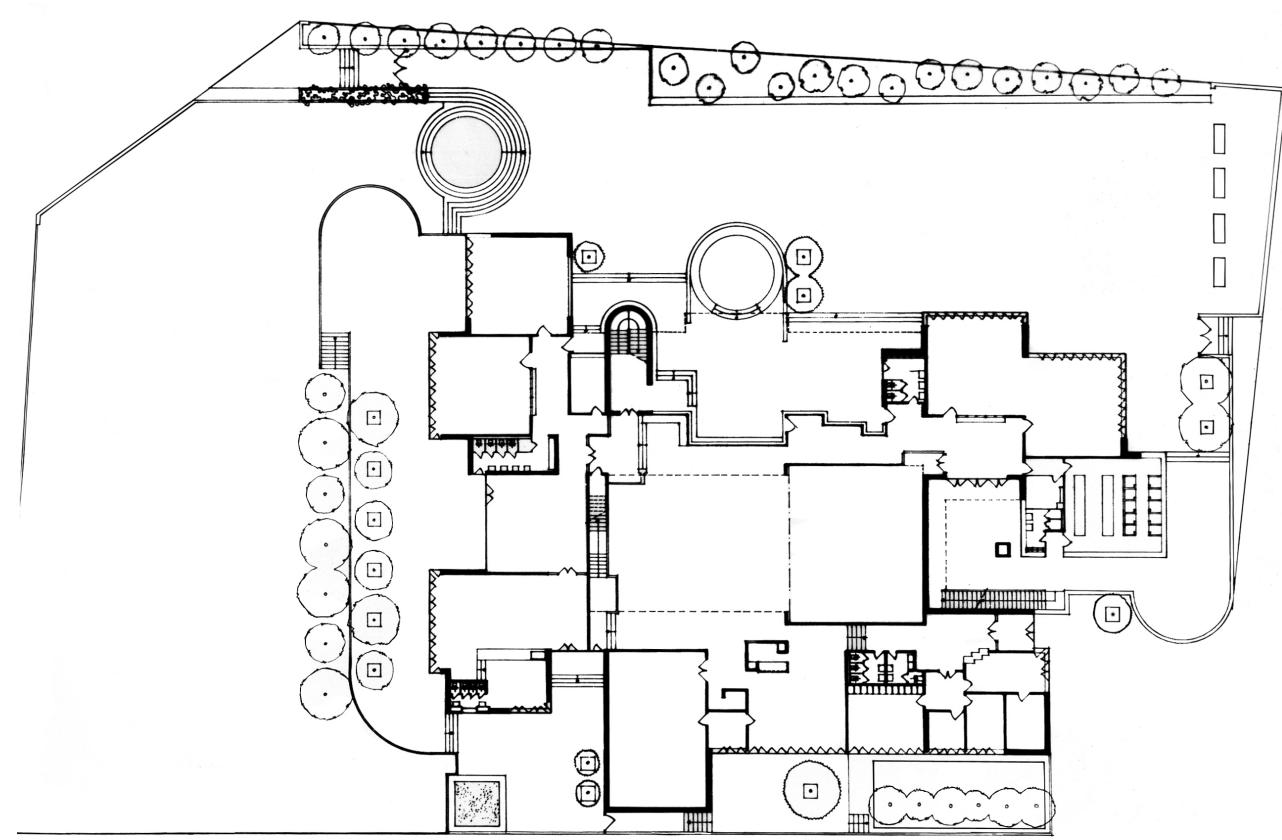

10

Nueva Forma (n. 86, 1973); y en las extranjeras Architecture Plus (Nueva York, n. 1, 1973) y L'Architecture d'Aujourd'hui (París, n. 177, 1975). Cabe destacar especialmente su publicación en Alemania, en el libro Schule im wandel / Wandel im Schulbau, que la situaba entre una selección de escuelas ejemplares de todo el mundo ${ }^{12}$.

En 1972, Martorell, Bohigas y Mackay volvieron a emplear el esquema centralizado en la escuela Thau, en Barcelona, pero introdujeron cambios sustanciales, derivados del desarrollo técnico y las nuevas orientaciones pedagógicas, que dejaban atisbar nuevas posibilidades para la arquitectura escolar. El estudio catalán accedió al proyecto a partir de un concurso restringido convocado por una entidad privada, Finestrelles S. A. Esta escuela debía incluir parvulario para 360 alumnos, enseñanza primaria para 720 y secundaria para 360; suponía la escuela de mayor capacidad que habían abordado. El proyecto debía atender a la reciente Ley General de Educación y ajustarse a los criterios del Ministerio de Educación y Ciencia para obtener una subvención pública.

En la escuela Thau se decidió diferenciar la enseñanza primaria de la secundaria en edificios independientes, lo que permitía controlar la escala del centro a pesar de su extenso programa. La pendiente pronunciada del terreno obligó a situar los edificios con una diferencia de nivel de $6 \mathrm{~m}$; no obstante, se dispuso un anfiteatro al aire libre entre ellos que servía de unión y de aglutinante de la vida colectiva del conjunto, mecanismo similar al de la escuela

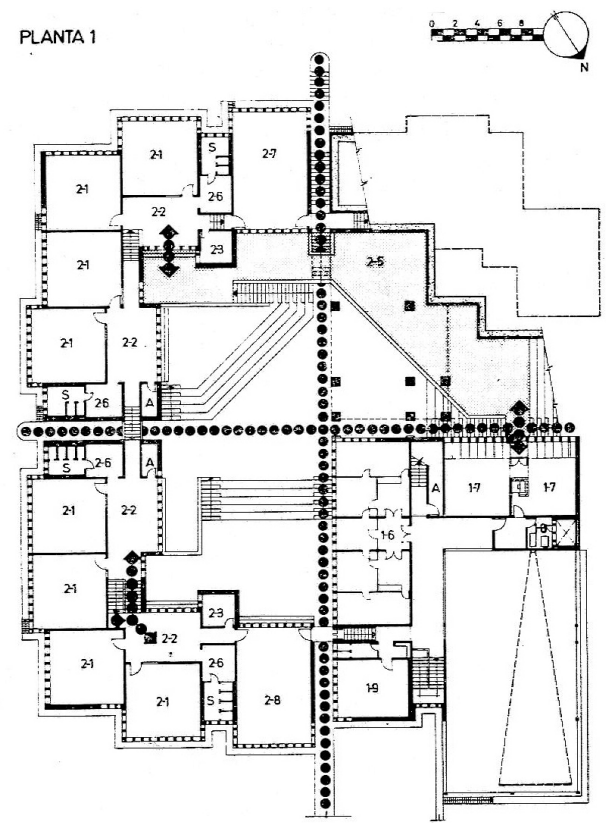

11

Fig. 9. Imágenes de la escuela Sant Jordi de Vilanova i la Geltrú: exterior y galería (material original facilitado por MBM Arquitectos).

Fig. 10. Planta de la escuela Sant Jordi de Vilanova i la Geltrú (material original facilitado por MBM Arquitectos).

Fig. 11. Planta de la escuela Sant Jordi del barrio de Montbau de Barcelona (Cuadernos de Arquitectura y Urbanismo, n. 89, 1972, p. 49).
12. A.A.V.V., Schule im wandel / Wandel im Schulbau, Gerd Hatje Forum Verlag, Stuttgart, 1975, pp. 52-53. 


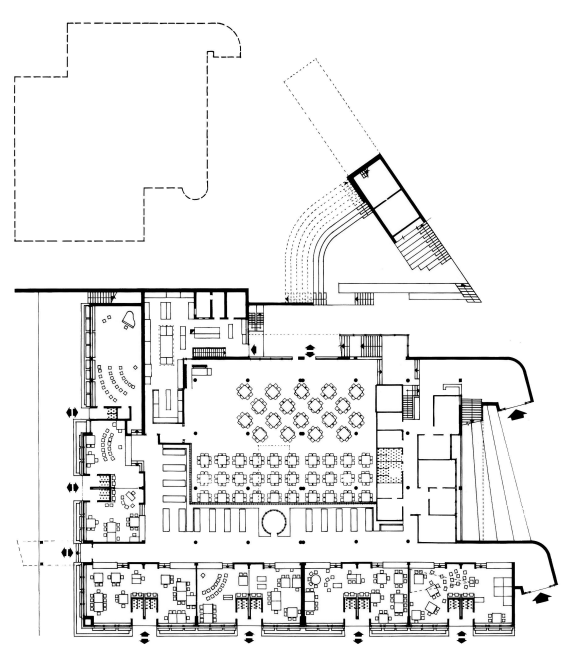

Fig. 12. Plantas de la escuela Thau en Barcelona según el proyecto definitivo (material original facilitado por MBM Arquitectos)

Fig. 13. Imágenes de la escuela Thau en Barcelona: anfiteatro al aire libre, fachada y escalera interior (material original facilitado por MBM Arquitectos).
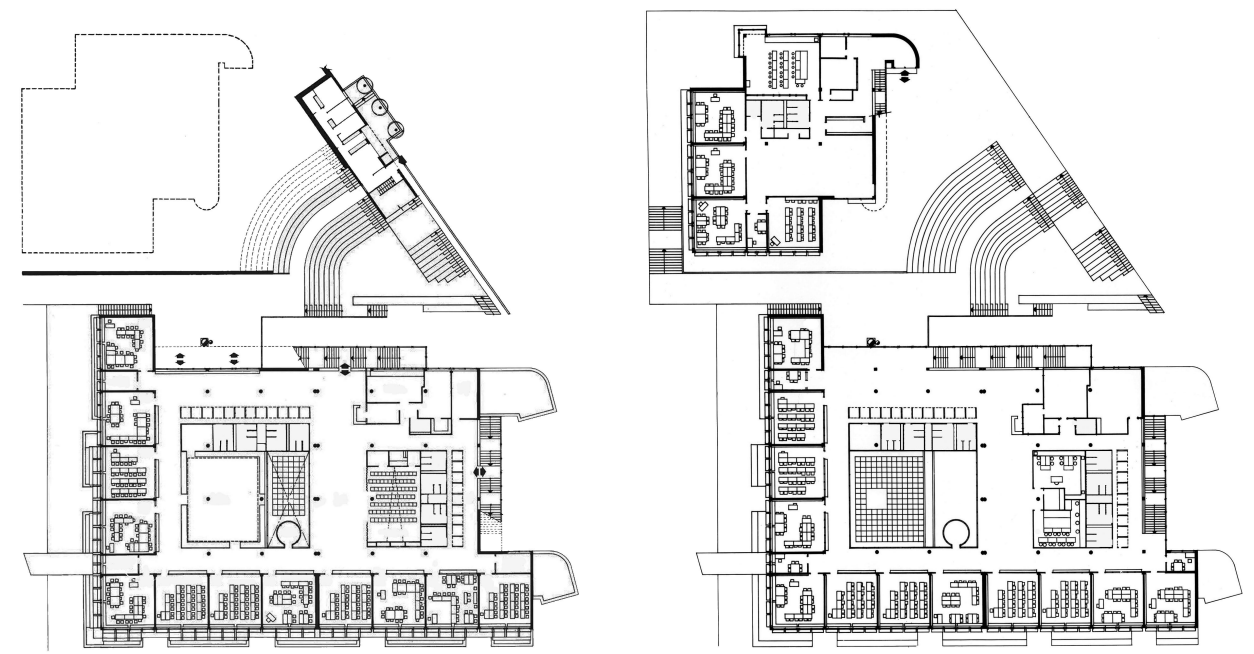

12
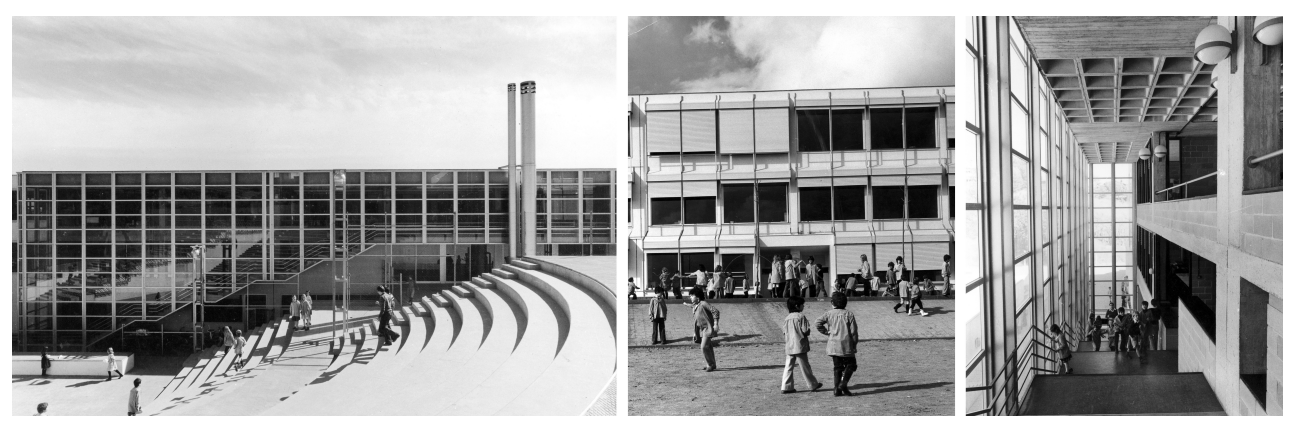

13

Sant Jordi en Montbau. A su vez, cada edificio poseía un espacio interior central rodeado de aulas orientadas a sureste y suroeste, se trataba por tanto de dos edificios concentrados. En el edificio de enseñanza secundaria, la especificidad del programa hacía inviable la disposición de un único espacio polivalente y se optó por la disposición de distintas salas de uso común en la zona central de cada planta. La comunicación vertical se producía a través de una escalera continua que vinculaba visualmente los distintos niveles (Figs. 12 y 13).

En este caso, los arquitectos definieron edificios de líneas continuas y volúmenes prismáticos que contrastaban con sus escuelas previas de perímetro quebrado. La geometría empleada en la escuela Thau colaboraba también a aumentar la vinculación entre los distintos tipos de espacios. Asimismo, los materiales empleados eran distintos: el ladrillo se sustituía por bloques de hormigón, las fachadas estaban revocadas y pintadas de blanco y contaba con grandes superficies de vidrio y carpinterías de aluminio. Bohigas atribuía estos cambios estéticos a los avances técnicos de la época ${ }^{13}$ :

\footnotetext{
“...intentamos hacer lo mismo pero ya con una arquitectura en la cual la tecnología y la estética es más optimista, más cercana al posible realismo de los anteriores idealismos. En realidad acepta incluso unas tendencias estilísticas más mediterráneas que nórdicas. Más industriales que populares, como si ya fuésemos un país rico".
}

La escuela Thau también tuvo una importante repercusión mediática y apareció en un gran número de revistas. En España fue publicada en Nueva Forma (n, 83-86, 1973), Cuadernos de Arquitectura (n. 111, 1975) y Arquitecturas Bis (n. 13-14, 1976). En el extranjero se publicó en The Architectural Review (Londres, n. 961, 1977), A+U Architecture and Urbanism (Tokio, n. 76, 1977), Wonen TA/BK (Amsterdam, n. 8, 1979), Controspazio (Roma, n. 4, 1979), L'Architecture d'Aujourd'hui (París, n. 204, 1979) y Werk Archithese (Zürich, n. 35-36, 1979), entre otras. Cabe destacar también la publicación de esta obra en el libro de Herman Hertzberguer: Space and Learning (Rotterdam, 010 Publishers, 2008). En ella, la escuela Thau sirve para ilustrar un capítulo dedicado a "La escuela como micro-ciudad", concepto que define a la perfección el propósito de sus autores ${ }^{14}$.
13. BOHIGAS, Oriol, Oriol Bohigas: realismo, urbanidad fracasos, cit. 


\section{TEMAS DE ARQUITECTURA ESCOLAR}

En 1972, la revista Cuadernos de Arquitectura y Urbanismo dedicó dos números consecutivos a la arquitectura escolar. Martorell, Bohigas y Mackay tuvieron especial participación en el segundo de ellos: sus obras sirvieron para ilustrar el desarrollo del edificio escolar en los últimos quince años y se incluyeron artículos de Oriol Bohigas y David Mackay. Ese mismo año, la revista publicó una separata dedicada en su totalidad a la arquitectura escolar del estudio catalán. En ella se incluyeron sus obras y artículos publicados en la revista y se añadieron otros textos realizados ex profeso. Estas publicaciones propiciaron que su trabajo incidiese de manera significativa en el devenir de la arquitectura escolar de la época.

La publicación monográfica manifestaba la actitud crítica y reflexiva que acompañaba a la labor práctica del estudio catalán. Los artículos de los tres arquitectos rebelaban un particular interés por el tema y amplios conocimientos al respecto. Así, sus textos recogían un conocimiento detallado de la tradición educativa catalana, de las experiencias pioneras internacionales y de las necesidades contemporáneas; bagaje fundamental en el desarrollo de sus edificios. También mostraban su interés por otras disciplinas relacionadas como la pedagogía. Cabe destacar especialmente su consciencia clara de los intensos cambios que estaba experimentando el edificio escolar a nivel internacional.

En "La evolución de la tipología escolar en Cataluña" Martorell repasaba el desarrollo de la escuela primaria en el siglo XX, de acuerdo con los sucesivos impulsos pedagógicos e ideológicos que habían incidido en Cataluña ${ }^{15}$. Para ello, recorría las etapas de evolución de las construcciones escolares y las iniciativas de edificación, con un especial acento en el caso de Barcelona. El texto exponía con detenimiento la etapa comprendida entre el comienzo de siglo y el inicio de la dictadura y extendía su periodo de análisis hasta los años 70. De esta manera, Martorell dibujaba una línea de continuidad entre los antecedentes de principio de siglo y su trabajo reciente ${ }^{16}$.

Por otro lado, los arquitectos estaban al corriente de las experiencias pioneras en la materia en otros países. Oriol Bohigas ha señalado que estar enterado de lo que pasaba en el mundo era algo natural, a pesar de las limitaciones del momento, era una tarea propia de cualquier profesional responsable ${ }^{17}$. Así, el arquitecto catalán, en su artículo "La escuela viva: un problema arquitectónico", sintetizaba de manera brillante la historia reciente del edificio escolar y demostraba un conocimiento exhaustivo de la actualidad internacional ${ }^{18}$. Además, David Mackay expuso en profundidad la experiencia pionera de Inglaterra en edificación escolar que, como se ha señalado, supuso una referencia constante en sus obras ${ }^{19}$.

Los artículos incluyeron, además de las escuelas inglesas, referencias a ejemplos notables de otros países como la escuela Bornheimer Hang en Frankfurt de Ernst May (1927), la escuela en Amsterdam de Jan Duiker (1930), la escuela en Alstetten de Alfred Roth (1933), la escuela Crow Island en Winetka de Saarinen, Perkins, Wheler y Will (1940), la guardería San'Elia en Como de Giuseppe Terragni (1936-1937), el proyecto de escuela en Darmstadt de Hans Scharoun (1951), las escuelas en Hilbersum de Willem Dudok (1920) y la escuela Millbrae en California (1962). También destacaban las referencias a monografías de arquitectura escolar: Edifici per l'instruzione, de Pasquale Carborana (1947), y The New School, de Alfred Roth (1950). Los arquitectos reconocían estas obras como referencias y Bohigas, de manera particular, ha destacado el valor de este depósito intelectual en la práctica de la arquitectura ${ }^{20}$.

Además, el conocimiento de las nuevas tendencias en educación y experiencias internacionales les sirvió para adelantar en su monografía nuevas posibilidades sobre el futuro del edificio escolar. El estudio catalán consideraba reveladora la experiencia de la construcción de escuelas en California, Estados Unidos, según el método de 'enseñanza programada', que perseguía la educación personalizada. Mackay citaba también en esta línea la experiencia inglesa de las 'escuelas abiertas', en las que el aula convencional dejaba paso a áreas caracterizadas dentro de un espacio único. En este nuevo modelo escolar el aula perdía su utilidad tradicional, se diluían totalmente los limites entre los distintos espacios y la escuela se transformaba en una única sala. Martorell creía posible la llegada de esta práctica didáctica a Cataluña y, con ella, una nueva etapa para el edificio escolar.
14. Kenett Frampton ha relacionado la escuela Thau con las de Herman Hertzberguer construidas en los 60 en Holanda. Cfr. FRAMPTON, Keneth, Martorell, Bohigas, Mackay: 30 años de arquitectura 1954-1984, op. cit., p. 20.

15. MARTORELL, Josep María, "La evolución de la tipología escolar en Cataluña", op. cit.

16. Los arquitectos tendrían muy presente su tradición. Incluso, Bohigas dio a conocer en el extranjero a los maestros de la arquitectura catalana en publicaciones como: "Luis Domenech y Montaner 1850-1923", Architectural Review, n. 849, noviembre de 1967, pp. 427-436.

17. Entrevista a Oriol Bohigas, en el estudio MBM Arquitectos, Barcelona, 3 de noviembre de 2011. Cabe añadir a este respecto que Oriol Bohigas mantuvo correspondencia durante esos años con destacados intelectuales catalanes y arquitectos de prestigio a nivel nacional e internacional. Cfr. BOHIGAS, Oriol, Epistolario, 1951-1994, cit.

18. BOHIGAS, Oriol, "La Escuela Viva: un problema arquitectónico", cit.

19. MACKAY, David, "Informe desde Inglaterra", Cuadernos de Arquitectura y Urbanismo, n. 89, 1972, pp. 50-61.

20. BOHIGAS, Oriol, Oriol Bohigas: Realismo, urbanidad y fracasos, cit. 
No obstante, este método pedagógico no llegó a extenderse y la escuela de espacio único mostró grandes inconvenientes en la práctica de la enseñanza tradicional. La continuidad espacial en estos edificios se reveló como un problema insalvable, debido a las interferencias que se daban entre los distintos grupos de alumnos, y los maestros - de formación tradicional- mostraron resistencia hacia las nuevas prácticas docentes y exigieron la compartimentación de los edificios. Incluso, la escuela Thau, de gran transparencia pero con una postura menos radical, encontró inconvenientes en su puesta en práctica derivados de su continuidad visual. Este suceso pone de manifiesto la estrecha vinculación entre las prácticas pedagógicas y la arquitectura en este tipo de edificios.

\section{CONCLUSIONES}

En suma, cabe destacar el trabajo de los arquitectos en materia escolar cuya intensa experimentación en los años 60 consiguió un modelo de espacios encadenados rico en matices con una gran capacidad didáctica y especial énfasis social. Martorell, Bohigas y Mackay emplearon en estas escuelas un esquema concentrado que fueron perfeccionando en los sucesivos proyectos y adaptando a las nuevas demandas educativas. El empleo de un modelo de analogía urbana les permitió incorporar la condición social de la escuela como oportunidad didáctica. Por un lado, estas escuelas contaban con espacios diferenciados acordes a la actividad educativa que albergaban $y$, a su vez, presentaban una vinculación fluida entre sus distintas partes.

Sus escuelas suponen uno de los ejemplos destacados realizados en España en los años 60 e, incluso, a nivel internacional. En ese momento, las nuevas tendencias educativas demandaron por primera vez escuelas de gran tamaño y con programas complejos que incorporaban distintas salas especializadas. En consecuencia, el control de la escala en estos centros de gran tamaño supuso un reto para los arquitectos, que debían evitar edificios masivos y conservar una escala contenida acorde al niño. En este contexto, las escuelas del estudio catalán destacaron como solución notable, ya que conseguían tanto minimizar el impacto del edificio en la ciudad como matizar espacios de distinta escala en su interior.

El acierto de estos proyectos estuvo relacionado con la actitud comprometida del estudio catalán. Los textos de Martorell, Bohigas y Mackay dedicados a la arquitectura escolar mostraron un particular interés por el tema y un amplio conocimiento al respecto. $\mathrm{Su}$ obra escolar se vio enriquecida por la tradición educativa catalana, las experiencias pioneras internacionales y los avances de la pedagogía. Los arquitectos concretaron proyectos de arquitectura escolar destacados que lograron un amplio reconocimiento profesional. La difusión de su trabajo a través de publicaciones especializadas les sirvió para convertirse en referencia e incidir en la importante transformación que experimentó el edificio escolar en el mundo en los años 60. vestigación posdoctoral en el Instituto de Ciencias Humanas, Sociales y Ambientales (INCIHUSA) del Consejo Nacional de Investigaciones Científicas y Técnicas (CONICET), Mendoza, Argentina. Su trabajo de investigación se enmarca en la historia de la arquitectura del siglo XX, aborda principalmente los edificios escolares y ha sido presentado en eventos de carácter internacional y publicado en revistas especializadas. De 2008 a 2013 ejerció la docencia en la Universidad de Navarra y, desde 2014, es profesora titular en la Universidad de Congreso de Mendoza. 